\title{
Feasibility, acceptability and uptake rates of gestational diabetes mellitus screening in primary care vs secondary care: findings from a randomised controlled mixed methods trial
}

\author{
Marie Tierney $^{1}$ - Angela O'Dea ${ }^{1}$ - Andriy Danyliv ${ }^{2}$ Liam G. Glynn ${ }^{3,4}$. \\ Brian E. McGuire ${ }^{4,5} \cdot$ Louise A Carmody $^{4} \cdot$ John Newell $^{6}$ • Fidelma P. Dunne ${ }^{1,4}$
}

Received: 23 April 2015 / Accepted: 3 July 2015 / Published online: 5 August 2015

(C) Springer-Verlag Berlin Heidelberg 2015

\begin{abstract}
Aims/hypothesis It is postulated that uptake rates for gestational diabetes mellitus (GDM) screening would be improved if offered in a setting more accessible to the patient. The aim of this study was to evaluate the proportion of uptake of GDM screening in the primary vs secondary care setting, and to qualitatively explore the providers' experiences of primary care screening provision.

Methods This mixed methods study was composed of a quantitative unblinded parallel group randomised controlled trial and qualitative interview trial. The primary outcome was the proportion of uptake of screening in both the primary and secondary care settings. All pregnant women aged 18 years or over, with sufficient English and without a diagnosis or diabetes or GDM, who attended for their first antenatal
\end{abstract}

Electronic supplementary material The online version of this article (doi:10.1007/s00125-015-3713-6) contains peer-reviewed but unedited supplementary material, which is available to authorised users.

Marie Tierney

marie.m.tierney@nuigalway.ie

1 School of Medicine, National University of Ireland, Galway, Ireland

2 J. E. Cairnes School of Business and Economics, National University of Ireland, Galway, Ireland

3 Discipline of General Practice, School of Medicine, National University of Ireland, Galway, Ireland

4 Galway Diabetes Research Centre, National University of Ireland, Galway, Ireland

5 School of Psychology, National University of Ireland, Galway, Ireland

6 HRB Clinical Research Facility, National University of Ireland, Galway, Ireland appointment at one of three hospital sites along the Irish Atlantic seaboard were eligible for inclusion in this study. Seven hundred and eighty-one pregnant women were randomised using random permutated blocks to receive a $2 \mathrm{~h}$ $75 \mathrm{~g}$ OGTT in either a primary $(n=391)$ or secondary care $(n=390)$ setting. Semi-structured interviews were conducted with 13 primary care providers. Primary care providers who provided care to the population covered by the three hospital sites involved were eligible for inclusion.

Results Statistically significant differences were found between the primary care $(n=391)$ and secondary care $(n=390)$ arms for uptake ( $52.7 \%$ vs $89.2 \%$, respectively; effect size 36.5 percentage points, $95 \%$ CI $30.7,42.4 ; p<0.001$ ), crossover ( $32.5 \%$ vs $2.3 \%$, respectively; $p<0.001)$ and nonuptake ( $14.8 \%$ vs $8.5 \%$, respectively; $p=0.005)$. There were no significant differences in uptake based on the presence of a practice nurse or the presence of multiple general practitioners in the primary care setting. There was evidence of significant relationship between probability of uptake of screening and age $(p<0.001)$. Primary care providers reported difficulties with the conduct of GDM screening, despite recognising that the community was the most appropriate location for screening.

Conclusions/interpretation Currently, provision of GDM screening in primary care in Ireland, despite its acknowledged benefits, is unfeasible due to poor uptake rates, poor rates of primary care provider engagement and primary care provider concerns.

Trial registration http://isrctn.org ISRCTN02232125

Funding This study was funded by the Health Research Board (ICE2011/03)

Keywords GDM - Gestational diabetes mellitus $\cdot$ Mixed methods $\cdot$ Primary care $\cdot$ Screening $\cdot$ Uptake rates 


\section{Abbreviations}

GDM Gestational diabetes mellitus

GP General practice/general practitioner

IPA Interpretative phenomenological analysis

\section{Introduction}

Gestational diabetes mellitus (GDM) is defined as 'any degree of glucose intolerance with onset or first recognition during pregnancy' [1]. The prevalence of GDM is believed to be increasing, predominantly due to increasing maternal age at pregnancy and increasing maternal obesity [2]. GDM prevalence has recently been reported as $2-6 \%$ in Europe [3], $9.3-25.5 \%$ internationally [4] and $12.4 \%$ in Ireland [5]. The condition has consequences for both maternal and fetal health, including macrosomia, increased risk of Caesarean section and increased neonatal unit admission [6-8]. In addition, a history of GDM is associated with a higher long-term risk for the development of impaired glucose tolerance, impaired fasting glucose and type 2 diabetes mellitus in the mother and her offspring [9-11].

Given this increasing prevalence, coupled with the potentially serious consequences of the condition, improving compliance with invitation to screening is of extreme importance to ensure identification and necessary treatment. However, a universal screening research study in Ireland showed only a $44 \%$ screening uptake rate [5]. A follow-up investigation indicated that uptake of screening was significantly influenced by the distance an individual must travel to the screening site [12]. Cullinan et al [12] postulated that overall screening uptake rates would increase by $6.6 \%$ if testing were to be moved from a regional hospital site to a more localised setting. Thus, implementing a screening programme that is more accessible is hypothesised to result in an improved screening uptake rate, thereby enabling the identification, treatment and reduction of the effects of GDM in a greater number of women. However, to the authors' knowledge, comparison of a primary vs secondary care-based universal screening programme for GDM has never been investigated.

Thus, the aims of this mixed methods study were to evaluate the rate of uptake of GDM screening in the primary vs secondary care setting, to examine potential factors affecting uptake rates and to examine the experience of provision of GDM screening in the primary care setting from the perspective of the service provider.

\section{Methods}

\section{Quantitative component}

This was an unblinded, parallel group, randomised controlled trial. The study was approved by the Galway University
Hospitals Research Ethics Committee, and all participants provided informed consent. Women who attended for their first antenatal appointment (typically at 12-18 weeks gestation) at one of three hospital sites along the Irish Atlantic seaboard between January 2013 and December 2013 were contacted by letter, followed up by a telephone call to invite them to participate in this research study. This recruitment strategy encompassed the invitation of all pregnant women in the region. Women who had a diagnosis of established diabetes or GDM were excluded, as were those under the age of 18 years and those who did not have a standard of English sufficient to provide informed consent. The three hospital sites included in this study involved a geographical area of approximately $10,000 \mathrm{~km}^{2}$ and served a catchment of approximately 500,000 people in both urban and rural populations. This included Galway city, the third largest city in the Republic of Ireland. Thus, the patients included in this study can be considered as broadly representative of the wider population of Ireland.

On the basis of a comparison of two proportions, a sample of 784 was deemed necessary to have $80 \%$ power, at the $5 \%$ significance level, to detect a $\geq 10 \%$ change in screening uptake assuming that a hospital-based screening programme delivered a screening uptake of $44 \%$. The randomisation process used random permutated blocks to ensure similar numbers of participants in each arm throughout the trial and equal numbers in each arm by the end of the study. Blocks of varying length were used to reduce the predictability of the allocation sequence. Prior to participant recruitment, an independent researcher generated the allocation sequence using the NQuery statistical software program version 2.0 (Statistical Solutions, Cork, Ireland; www.statistical-solutions-software. com) and used sealed envelopes to ensure that the allocation sequence was concealed from study researchers until the intervention group was assigned.

The protocol for this study has been previously published [13]. Seven hundred and eighty-one women consented to participation after written and telephone communication. Each woman consented to a $2 \mathrm{~h}, 75$ g OGTT between 24 and 28 weeks gestation and was randomised to either the primary or secondary care screening group. When a woman received the test in the alternate screening location to which she was allocated, she was deemed to have crossed over study arms. The flow of participants through the trial is displayed in Fig. 1.

All women randomised to the primary care group were offered an appointment for a GDM screen at their general practitioner (GP) clinic. All women randomised to the secondary care group were offered a screening test appointment at the hospital at which they were receiving antenatal care.

Analysis was conducted by intention to treat. The primary outcome was the proportion of uptake of screening in both the primary and secondary care settings. This was measured as the proportion of the offers accepted from the total number of 
Fig. 1 Flow chart of participant enrolment, randomisation and analysis

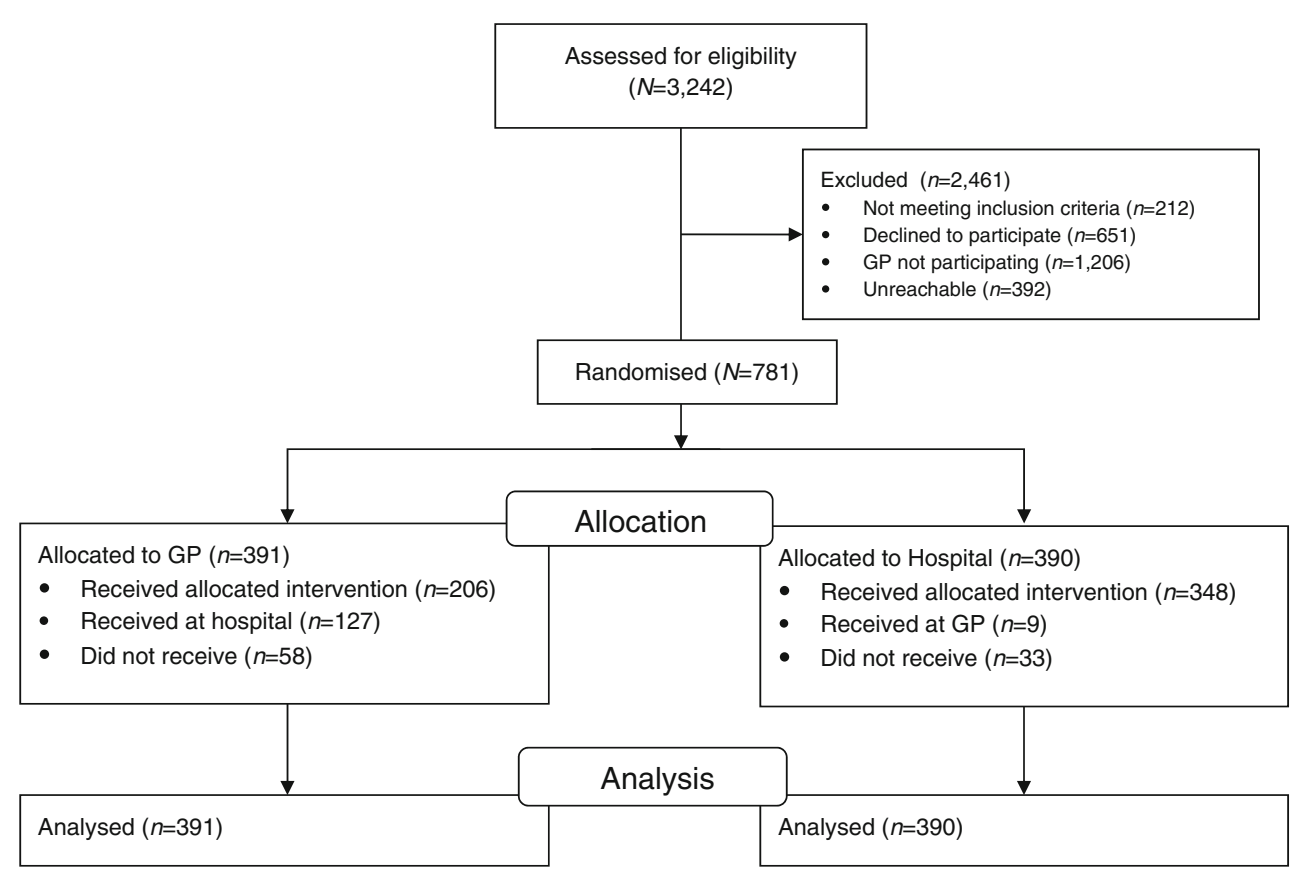

screens offered for each location. The frequency and proportion of uptake in the primary and secondary setting were calculated. The difference in uptake proportions and corresponding $95 \%$ CIs were calculated using an exact test for a twosample comparison of binomial responses. In the primary care setting, the $\chi^{2}$ test for independence was used to determine whether there was an association between screening uptake at the randomised location based on the following criteria: (1) the presence or absence of a practice nurse and (2) the existence of a solo GP or multiple GP practice. A nominal logistic regression model and classification tree analysis was used to estimate the probability of uptake of screening at the randomised screening location, adjusting for age, BMI, previous GDM and family history of diabetes mellitus. All analyses were carried out using Minitab 17 (Minitab, State College, PA, USA), R version 3.02 and SPSS version 20 (SPSS, Chicago, IL, USA).

\section{Qualitative component}

This aspect of the study involved semi-structured interviews with a selection of primary care providers who either participated fully, dropped out of the trial or did not agree to participate in the trial. Thirteen primary care providers (11 GPs, two practice nurses) were purposively sampled and agreed to participate in this aspect of the study. The research questions covered the primary care provider's experiences of involvement in the trial and their opinions on the future provision of GDM screening.

A semi-structured interview guide was used in this study, with primarily open-ended questions to elicit as much information on respondents' experiences as possible. A conversational style of interviewing was adopted to encourage a comfortable and fluent dialogue, while using an interview schedule as a reference to ensure that key topics were covered. The first and second authors performed all interviews via telephone, at a time of the participants choosing. Interviews lasted 10-20 min.

The interviews were audio-taped, with the permission of each participant, using a digital recorder and were then transcribed verbatim. The transcripts of the interviews were analysed thematically using interpretative phenomenological analysis (IPA) [14]. IPA focuses on the exploration of participants' experience, understandings, perceptions and views in order to explore the processes through which participants make sense of their own experiences. The three stages of analysis involve: (1) reading and re-reading each transcript and recording some observations and reflections; (2) transforming these initial notes into emerging themes and (3) clustering these emerging themes based on conceptual similarities. At each stage, quotations illustrating each theme were documented. In line with the recommendations of Elliot et al [15], 'credibility checks' were undertaken to ensure that the themes accurately reflected the data.

\section{Results}

\section{Quantitative results}

There were 3,242 potential participants for this study. A total of 1,206 individuals (37.2\%) were not contacted as their primary care provider did not wish to participate in the research study. A total of 212 participants did not meet the inclusion 
criteria, 651 declined to participate and 392 were unreachable after written communication and three telephone call attempts.

In total, 781 women $(24.1 \%)$ were recruited to this study and randomised to have screening completed either in the primary care setting $(n=391)$ or the secondary care setting $(n=390)$. Baseline characteristics of the study participants are shown in Table 1.

There was evidence of a significant association between randomised location and proportion of screening uptake (Fig. 2). Of those randomised to have screening conducted in primary care, the proportion of screening uptake was $52.7 \%$ $(n=206)$ while for those randomised to have screening in secondary care, the proportion of screening uptake was $89.2 \%$ $(n=348)$ (Fig. 2). This difference of 36.5 percentage points was statistically significant ( $p<0.001 ; 95 \%$ CI 30.7, 42.4).

Almost one-third of the women $(n=127 ; 32.5 \%)$ who were randomised to the primary care group had the test completed in the secondary care setting. Conversely only $2.3 \%(n=9)$ of those randomised to the secondary care setting had the test completed in the primary care setting (Fig. 2). This difference of 30.2 percentage points in crossover between randomised site and actual screening site was statistically significant $(p<0.001 ; 95 \%$ CI 25.3, 35.0).

When assessing those who did not attend for screening at all, $14.8 \%(n=58)$ of those randomised to primary care did not attend for screening compared with $8.5 \%(n=33)$ of those randomised to secondary care (Fig. 2). This difference of 6.4 percentage points was statistically significant $(p=0.005 ; 95 \%$ CI $1.9,10.8)$.

There was no evidence of a significant difference in the proportion of uptake of screening at the randomised location in the primary care group $(n=391)$ based on the presence of a practice nurse $(p=0.155)$ or the presence of multiple GPs in a practice $(p=0.921)$.

When examining the probability of uptake of screening, there was evidence $(p<0.001)$ of a significant relationship with age (Fig. 3). For those that were randomised to be screened in the primary care setting, the probability of uptake of screening or crossover increased with age; the probability of no uptake of screening was higher for younger participants but decreased with increasing age.

Table 1 Baseline characteristics of study participants

\begin{tabular}{lll}
\hline Characteristic & $\begin{array}{l}\text { Primary care } \\
(n=391)\end{array}$ & $\begin{array}{l}\text { Secondary care } \\
(n=390)\end{array}$ \\
\hline Age, years, mean (SD) & $25.5(4.7)$ & $25.7(5.1)$ \\
BMI, kg/m ${ }^{2}$, mean (SD) & $32.8(5.4)$ & $33.1(4.9)$ \\
Family history of diabetes, $n(\%)$ & $55(14.1)$ & $68(17.4)$ \\
Previous GDM, $n(\%)$ & $1(0.3)$ & $7(1.8)$ \\
\hline
\end{tabular}

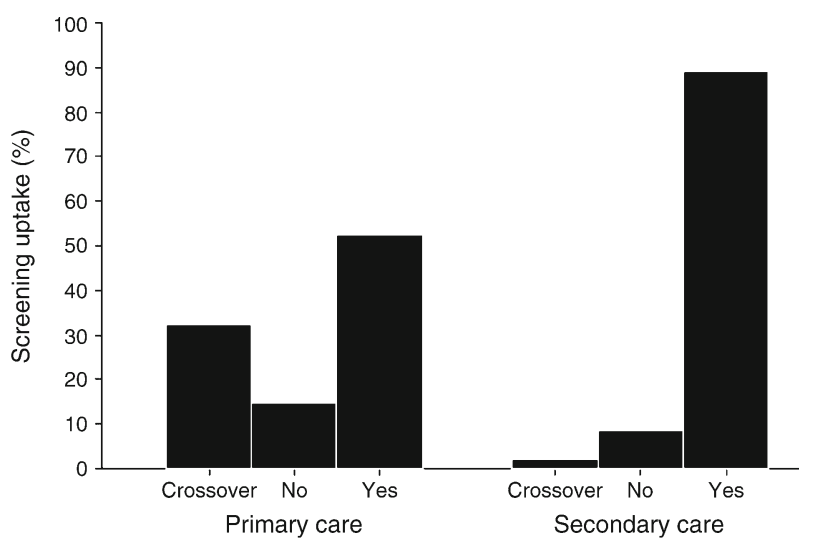

Fig. 2 Screening uptake by randomised screening location. $p<0.001\left(\chi^{2}\right.$ test)

For those that were randomised to the secondary care setting, there was a low probability of crossover regardless of age. The probability of uptake of screening was high and increased further with increasing age. The probability of no uptake of screening decreased with increasing age.

\section{Qualitative results}

The analysis of the interviews with the primary care providers yielded four central themes. The first three themes related to the primary care providers' perceptions of the feasibility of GDM screening in the primary care setting. The last theme related to their perceptions of the appropriateness of GDM screening in primary care. Direct quotations relevant to each of the themes are provided in the electronic supplementary material (ESM) text. a

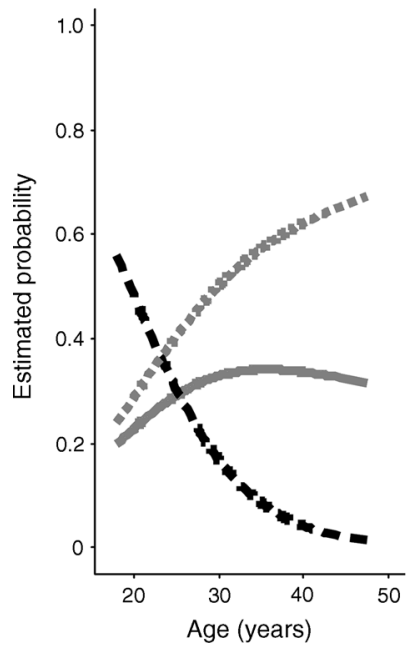

b

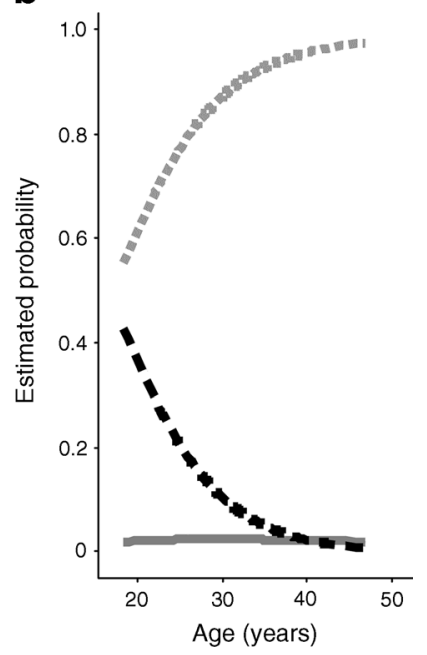

Fig. 3 (a) Estimated probability of screening uptake at the primary care setting by location and age. (b) Estimated probability of screening uptake at the secondary care setting by location and age. Grey dashed line, Yes; grey continuous line, Crossover; black line, No 
Theme 1: workload barriers Primary care providers described the workload associated with the provision of the OGTT as a barrier to the feasible provision of GDM screening in their practices. One of the key concerns relating to the workload revolved around the necessity for three blood draws in the OGTT and the time taken to complete these tasks.

Many primary care providers made reference to a practice nurse being better placed than a GP to administer the test and in most cases it was the practice nurse who administered the OGTTs in practices that employed a practice nurse. Practices not employing a nurse found participation in this trial particularly difficult.

Theme 2: logistical barriers The logistical difficulties posed by the provision of GDM screening in primary care and the disruption to practice this caused were also reported as barriers to screening.

Primary care providers described GP practices as being centres that dealt with patients at short notice. These patients often presented as higher clinical priority than a GDM screening patient, which made it difficult to carry out the test protocol in an accurate and timely fashion.

Similarly, because of the nature of the test protocol and the necessity of blood draws at precise times, the requirement that a woman undergoing GDM screening may have to bypass other waiting patients was considered to be a difficulty.

When probed about being able to maintain the precise schedule of blood draw timings, primary care providers were unsure if this could be met within their service.

Rural practices may be located at a considerable distance from the laboratory where the bloods are analysed. In those practices, blood work was only completed in the practice on specified days each week and samples were collected by courier for delivery to the laboratory at a specific time. The coordination of GDM screening within this framework proved logistically difficult for many rural practices.

Theme 3: financial barriers Another concern that primary care providers expressed regarding the feasibility of providing GDM screening in the primary care setting relates to the financial compensation which is received for the provision of the service.

Much of the concern about the financial remuneration appears to stem from a sense that more is being asked from primary care providers while their statutory payments are being cut simultaneously. Primary care providers appeared worried that GDM screening would be moved from secondary to primary care without any financial compensation being made. There also appears to be an overall sense of negativity and disillusionment among primary care providers presently, which may also be linked to their unwillingness to provide GDM screening
Theme 4: appropriateness of primary care screening Despite the barriers to the provision of GDM screening, there was overwhelming positivity about the benefits of GDM screening occurring in a localised community-based setting rather than in regional secondary care settings. The primary care providers acknowledged that provision in the primary care setting was particularly beneficial from the point of view of the patient. This is particularly relevant to patients living at a considerable distance from the hospital screening location.

Primary care providers were also aware that provision of screening in GP practices would facilitate patient follow-up, which they perceived as beneficial.

As primary care providers are cognisant of the benefits to the patient and the opportunity that the primary care setting affords for increased levels of combined follow-up care, a number of primary care providers proposed an alternative screening option that would allow for the benefits of provision by primary care providers to be retained but without the difficulties previously reported. This proposal involved the screening service being provided in the community by a dedicated individual external to the workings of their practice, either in a community health centre or in the GP practice.

\section{Discussion}

The quantitative results of this trial show significantly higher screening uptake rates in the secondary care setting than in the primary care setting and significantly lower crossover rates and lower non-uptake rates in the secondary care setting. Furthermore, almost one-third of potential participants were ineligible for this trial as their primary care providers were unwilling to provide GDM screening. The presence of a practice nurse or the existence of a multiple GP practice did not have an impact upon whether women randomised to the primary care setting for their screening appointment attended there. Age was the only risk factor assessed which showed a significant relationship with proportions of screening uptake.

The findings of this study are not consistent with the assertion by Cullinan et al [12] who suggested that there would be an increase in screening uptake rates of $6.6 \%$ if testing was moved from the secondary care setting to a more localised primary care setting. Our qualitative results indicate that the primary care providers encountered many practical difficulties, such as workload demands, logistical challenges and remuneration concerns, which made the undertaking of screening unfeasible and unsustainable for them and may, at least in part, explain the poor rates of uptake and poor engagement in the primary care setting.

Buckley et al [3] reported that screening practice and policy is inconsistent across Europe, and there is a lack of consensus on a variety of related issues. This includes the location in which antenatal care, including GDM screening, is provided. 
Countries like Ireland, Australia, the UK and USA generally have antenatal care that is directed by the hospital-based obstetrician [16]. Conversely, Scandinavian countries (Sweden, Norway, Denmark), the Netherlands, Canada and New Zealand typically provide routine antenatal care in the community [16-19], with midwives and GPs providing the care (the former to a larger degree). In these countries there does not appear to be resistance to the provision of such services by primary care providers. Conversely, the trial reported here encountered significant resistance from primary care providers.

There appears to be two distinct differences between the provision of this trialled screening protocol and the screening protocol currently provided by primary care in the aforementioned counties which provide un-resisted GDM screening: (1) the implementation of a new screening protocol during a time of reform in primary care and (2) the use of midwives in the provision of antenatal care.

This study is set in a time of extreme flux and uncertainty for Irish GPs. This was highlighted in the qualitative component of this study as well as by national organisations representing GPs. Ireland's primary care sector is currently undergoing a period of reform. This seems to be playing a role in the primary care providers' willingness to provide GDM screening and their perception of the feasibility of providing GDM screening in primary care.

A doctor's job satisfaction is particularly prone to change if conditions of work change [20], as would be assumed during times of reformative change. The professional satisfaction of doctors is a proven useful indicator of the good functioning of the healthcare system [20]. Furthermore, doctors' discontent is known to lead to undesirable outcomes such as lack of motivation [20] and poor patient care [21, 22]. It is reasonable to assume that the challenges posed by the currently implemented reform may be leading to job dissatisfaction among practitioners, resulting in a general unwillingness to engage in novel interventions, explaining why uptake in the primary care setting for the intervention trialled here was poorer than hypothesised. This has repercussions for the implementation of novel treatment methods in times of reform in other clinical situations nationally and internationally.

Qualitative analysis revealed that primary care providers felt that the presence of a practice nurse would make it easier and more feasible to provide screening in primary care. Midwives are the predominant health provider of antenatal care in those countries that provide antenatal care in the primary care setting [16-19]. Thus, it seems reasonable that primary care providers deemed their presence valuable in the effective provision of GDM screening in primary care. However, quantitative analysis of whether there was a difference in uptake at the primary care level between practices which had a practice nurse and those that did not demonstrated that this was not to be the case, indicating that those practices with a practice nurse had other significant barriers to GDM screening provision.
Overall, the proportion of women availing themselves of screening was high in comparison to that reported in other published work in Ireland. The overall uptake rate in the secondary care setting in this study was almost $90 \%$, greatly increased from the uptake rate of $44 \%$ in the secondary care setting found previously [5]. The previous work was completed between 2006 and 2009 and since that time there has been research into GDM occurring in the region $[5,23,24]$ as well as an educational campaign to increase awareness of GDM and promote screening. This may have contributed to the higher levels of overall uptake and highlights the long-term beneficial clinical impact that engagement in research can facilitate. In particular, the age of 30 years seems an important indicator of those who receive GDM screening. This may potentially be linked to educational campaigns [25] that have been put in place in the region and which highlight a maternal age of $>35$ years as a risk for development of GDM and stress the necessity for screening.

The qualitative findings, along with international perspectives, give us indications as to why, despite the Atlantic Diabetes in Pregnancy 1 study [12] suggesting significant improvements in uptake were likely as a result of offering screening in primary care, this was not shown to be the case. The results of this mixed methods research study appear to indicate that without adequate resourcing the primary care setting is not currently a feasible alternative to the hospital setting for conducting GDM screening. It appears likely that resistance to change during reform combined with the additional logistical and workload challenges that GDM screening imposes upon GPs in primary care limit its implementation currently. Countries not undergoing primary care reform and those with a strong nursing/midwifery component to primary care may be better able to implement this novel screening strategy and thus may experience better uptake rates in primary care than found in our trial.

The study has a number of strengths. To the authors' knowledge, this is the first study to assess the provision of GDM screening in the primary care setting and provides unique mixed methods data on this topic. The qualitative data provide an interesting insight into the experiences and concerns of primary care providers. This is very important as it helps to explain the low uptake of screening in primary care and raises serious questions about the feasibility of conducting screening in primary care in the absence of either increased resources for primary care providers or an alternative model of community-based screening. A further strength lies in the fact that we gained a balanced view of the perspectives of the primary care providers in the region by interviewing those who were willing to provide the GDM screening throughout the trial, those who provided only a small number of screens before withdrawing and those who were unwilling to be involved in the trial at all. 
There are some limitations of the study. The data collected are from three hospital sites in one region of the country and thus may not extrapolate accurately to the rest of the country or indeed internationally, where different service models are in operation. However, international perspectives have revealed that reformative times can lead to unwillingness to engage among practitioners and thus it can be assumed that the results of this study can be extrapolated to primary care services which are undergoing reformative measures. Furthermore, women who were insufficiently proficient in English to give informed consent were excluded from the study. This may have had an impact on the generalisability of the findings. Also, 37\% of women from the sample frame could not be invited to participate in the trial as their primary care providers were not willing to take part. Thus we do not know how their participation might have affected the outcomes.

Overall, this study identified an increase in GDM screening uptake rates when compared with uptake rates in 2006-2009 [5]. Although primary care providers perceived themselves to be better placed to provide GDM screening, uptake rates were significantly lower and crossover rates and non-uptake rates were significantly higher in the primary care setting when compared with secondary care.

Presently, there are many obstacles to a change in current practice that may be influencing the rates of uptake for those randomised to receive screening in primary care. Future research could evaluate different methods by which to incentivise primary care providers or alternative communitybased models.

The results of this mixed methods study appear to indicate that without adequate resourcing, primary care is not currently a feasible alternative to the secondary care setting for the conduction of GDM screening in this region of Ireland.

\begin{abstract}
Acknowledgements The material is based on work supported by the Health Research Board of Ireland. We would also like to acknowledge the assistance provided by the patients, hospital staff and primary care providers involved in this study, without which this trial would not have been possible. We would also like to acknowledge the reviewers of drafts of this manuscript, whose suggestions have strengthened the final version.
\end{abstract}

Funding This study was supported by a grant from the Health Research Board of Ireland (ICE/2011/3). The study sponsor was not involved in the design or conduct of the study, collection, management, analysis or interpretation of the data or the preparation, review or approval of the manuscript for publication.

Contribution statement MT had full access to all of the data in the study and takes responsibility for the integrity of the data and the accuracy of the data analysis. All authors meet ICJME uniform requirements for authorship, including substantial contributions to conception and design, acquisition of data or analysis and interpretation of data and drafting the article or revising it critically. All authors had final approval of the version to be published.
Duality of interest The authors declare that there is no duality of interest associated with this manuscript.

\section{References}

1. American Diabetes Association (2013) Diagnosis and classification of diabetes mellitus. Diabetes Care 36:S67-S74

2. Xiong X, Saunders L, Wang F, Demianczuk N (2001) Gestational diabetes mellitus: prevalence, risk factors, maternal and infant outcomes. Int J Gynecol Obstet 75:221-228

3. Buckley B, Harreiter J, Damm P et al (2012) Gestational diabetes mellitus in Europe: prevalence, current screening practice and barriers to screening. A review. Diabet Med 29:844-854

4. Sacks DA, Hadden DR, Maresh M et al (2012) Frequency of gestational diabetes mellitus at collaborating centers based on IADPSG consensus panel-recommended criteria: the Hyperglycemia and Adverse Pregnancy Outcome (HAPO) Study. Diabetes Care 35: 526-528

5. O'Sullivan E, Avalos G, O'Reilly M, Dennedy M, Gaffney G, Dunne F (2011) Atlantic Diabetes in Pregnancy (DIP): the prevalence and outcomes of gestational diabetes mellitus using new diagnostic criteria. Diabetologia 54:1670-1675

6. Catalano PM, McIntyre HD, Cruickshank JK et al (2012) The hyperglycemia and adverse pregnancy outcome study: associations of GDM and obesity with pregnancy outcomes. Diabetes Care 35: 780-786

7. Schmidt MI, Duncan BB, Reichelt AJ et al (2001) Gestational diabetes mellitus diagnosed with a 2-h 75 -g oral glucose tolerance test and adverse pregnancy outcomes. Diabetes Care 24:1151-1155

8. Langer O, Yogev Y, Most O, Xenakis EM (2005) Gestational diabetes: the consequences of not treating. Am J Obstet Gynecol 192: 989-997

9. Engeland A, Bjørge T, Daltveit AK et al (2011) Risk of diabetes after gestational diabetes and preeclampsia. A registry-based study of 230,000 women in Norway. Eur J Epidemiol 26:157-163

10. O'Sullivan JB (1991) Diabetes mellitus after GDM. Diabetes 40: 131-135

11. Kim C, Newton KM, Knopp RH (2002) Gestational diabetes and the incidence of type 2 diabetes a systematic review. Diabetes Care $25: 1862-1868$

12. Cullinan J, Gillespie P, Owens L, Dunne F (2012) Accessibility and screening uptake rates for gestational diabetes mellitus in Ireland. Health Place 18:339-348

13. O'Dea A, Infanti JJ, Gillespie P et al (2014) Screening uptake rates and the clinical and cost effectiveness of screening for gestational diabetes mellitus in primary versus secondary care: study protocol for a randomised controlled trial. Trials 15:1-9

14. Smith JA, Flowers P, Larkin A (2009) Interpretative phenomenological analysis: theory, method and research. SAGE, London

15. Elliott R, Fischer CT, Rennie DL (1999) Evolving guidelines for publication of qualitative research studies in psychology and related fields. Br J Clin Psychol 38:215-229

16. Rowland T, McLeod D, Froese-Burns N (2012) Comparative study of maternity systems. Ministry of Health, Wellington

17. Holan S, Mathiesen M, Petersen K (2005) A national clinical guideline for antenatal care. Directorate for Health and Social Affairs, Oslo

18. Persson M (2009) Gestational diabetes mellitus - experiences of pregnant women, midwives, and obstetricians and the performance of screening. Department of Clinical Science, Umea Univesitet, Umea 
19. Bernloehr A, Smith P, Vydelingum V (2005) Antenatal care in the European Union: a survey on guidelines in all 25 member states of the community. Eur J Obstet Gynecol Reprod Biol 122:22-32

20. Perneger TV, Deom M, Cullati S, Bovier PA (2012) Growing discontent of Swiss doctors, 1998-2007. Eur J Pub Health 22:478-483

21. Haas JS, Cook EF, Puopolo AL, Burstin HR, Cleary PD, Brennan TA (2000) Is the professional satisfaction of general internists associated with patient satisfaction? J Gen Intern Med 15:122-128

22. Quinn MA, Wilcox A, Orav EJ, Bates DW, Simon SR (2009) The relationship between perceived practice quality and quality improvement activities and physician practice dissatisfaction, professional isolation, and work-life stress. Med Care 47:924-928
23. Owens LA, O'Sullivan EP, Kirwan B, Avalos G, Gaffney G, Dunne F (2010) ATLANTIC DIP: the impact of obesity on pregnancy outcome in glucose-tolerant women. Diabetes Care 33:577-579

24. Noctor E, Crowe C, Carmody L et al (2013) ATLANTIC DIP: simplifying the follow-up of women with previous gestational diabetes. Eur J Endocrinol 169:681-687

25. Dunne F (2009) Clinical practices: guidelines for the management of diabetes in pregnancy. Atlantic DIP. Available at: http:// atlanticdipireland.com/wp-content/uploads/2010/03/Guidelinesfor-the-management-of-Diabetes-in-Pregnancy.pdf. Accessed 17 Nov 2014 